\title{
The Wetting Properties of Powders and Devices for Measuring Them
}

\author{
Noriyoshi Kaya \\ Micromeritics Laboratory, $R \& D$ Div. \\ Hosokawa Micron Corporation* \\ Masumi Koishi \\ Faculty of Industrial Science and Technology, \\ Science University of Tokyo**
}

\section{Introduction}

Once a solid comes into contact with liquid, a phenomenon known as "wetting" occurs. Wetting is an interfacial phenomenon where liquid molecules are adsorbed onto the surface of a solid. According to interfacial chemistry textbooks ${ }^{1)^{\sim 3)}}$, it is defined as a phenomenon where the gas-solid interface is disrupted, and a liquidsolid interface occurs.

This phenomenon appears responsible for the adhesion and the affinity of liquid molecules relative to a solid surface. Both the interaction between the liquid molecules and the solid surface can be evaluated by using this phenomenon. The wetting measurement technique is a long-established means ${ }^{4)}$ for evaluating the surface properties of various materials in which certain liquids penetrate. This technique has been basically studied, especially in fabrics technology 5 ) 7).

The many applications of wetting include at one extreme the laundering of clothes. Other applications are industrial bases, ore floatation, impregnation with insulator oil, the molding of plastics, and the dispersion of various pigments in liquids. Recently, applications such as liquid penetration into a paper filter for paper chromatography ${ }^{8)}$, and the preparation of composite materials by adjoining ceramics to metals have been found. In essence, this phenomenon has indispensable roles of modern technology.

The discussion in this paper is mainly about powders, especially on studies the measurement of wetting in Japan.

* No. 9, 1-chome, Shoudai Tajika, Hirakata, Osaka, 573 TEL. (0720) 55-2220

** 102-1, Oshamanbe-cho, Yamagoe, Hokkaido, 049-35 TEL. (01377) 2-5111

Received Sep. 30, 1988
In studying the measurement of the wetting of a powder, unlike that of solid matter, there are several technical difficulties that are not readily solved: data taken on a specific powder by one researcher may be different from that taken by another researcher; replicability of the wetting measurement of powders can be poor, and measurement values may be unstable. These problems are mainly attributable to the high surface energy of the powder, and partly to the number of uncertain factors in measuring the wetting of a powder bed. These factors include uncompacted structures within a powder bed, the presence of ultrafine particles, and uneven moisture content.

\section{Expression of wettability}

As previously defined, wetting is a phenomenon where the gas-solid interface is disrupted, and a liquid-solid interface occurs. A quantitative evaluation of this phenomenon has been discussed on the basis of the energy balance. The change in this energy is expressed in Fig. $1^{9)}$.

The change in the surface energy during wetting is analyzed into two relationships: one based on free energy, and the other based on total energy.

As shown in Fig. 2, a droplet of a liquid with good wetting, placed on the solid surface, produces a smaller angle of contact. The angle formed between the droplet and the solid surface is smaller, whereas the droplet of the liquid with poor wetting indicates a larger angle.

In this figure, $\gamma$ indicates surface tension, that represents the work necessary to form a unit area of the additional interface. It is expressed as the free energy of the phenomenon, where, the suffix $S$ represents the solid, and $L$, 


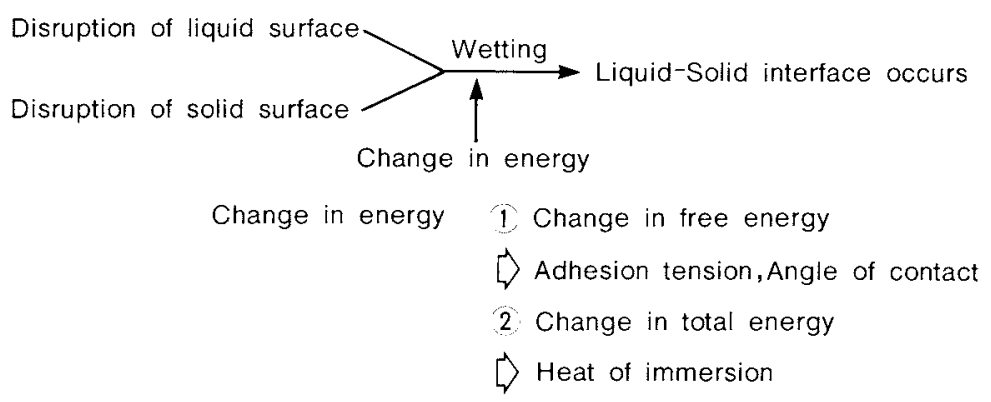

Fig. 1 Change in energy of the wetting process

the liquid.

Considering the balance of the horizontal component of the forces, the widely known Young's equation can be deduced as follows.

$$
\gamma_{S}=\gamma_{S L}+\gamma_{L} \cos \theta
$$

$\gamma_{L} \cos \theta$ in Eq. (1) is called the adhesive tension, which is equivalent to the free energy of a solid being immersed in a liquid.

For this reason, the adhesive tension is obtained by the measurement of a surface tension $\gamma_{L}$ of liquid and the angle of $\operatorname{contact} \theta$. Generally, however, a value $\theta$ is used to indicate the wettability of solid matter.

Generally speaking, once a liquid comes into contact with solid matter, heat generates even in the absence of a chemical reaction, caused by the interaction between the solid and the liquid in which the solid is immersed. When the enthalpy of the solid surface is $H_{S}$, and that of the liquid-solid interface is $H_{L S}$, the heat of immersion $Q$ is indicated as the difference between these enthalpies.

$$
Q=H_{L S}-H_{S}=\Delta H
$$

Furthermore, there is a correlation between the enthalpy and the total energy as shown below;

$$
\Delta H=\Delta E+p \Delta V
$$

Since the volumetric change $\Delta V$ can be negligible in the wetting process;

$$
\triangle H=E_{S L}-E_{S}=\Delta E
$$

The heat of immersion corresponds with the total energy. Under a constant pressure, the interrelationship between the total energy and the free energy gives the Gibbs-Helmholtz equation. Applying this equation to the wetting, the following equation can be obtained;

$$
\Delta E=\Delta \gamma-T\left[\frac{\partial(\Delta \gamma)}{\partial T}\right]_{P=\text { const }}
$$

Where the change $\Delta \gamma$ in the interfacial free energy is;

$$
\Delta \gamma=\gamma_{S}-\gamma_{S L}=\gamma_{L} \cos \theta
$$

Combining Eqs. (2), (4), (5), and (6);

$$
Q=\gamma_{L} \cos \theta-T\left[\frac{\partial\left(\gamma_{L} \cos \theta\right)}{\partial T}\right]
$$

Thus, the angle of contact $\theta$ can be obtained by the measurement of the heat of immersion $Q$, the surface tension of the liquid $\gamma_{L}$ and the estimation of $\partial \gamma_{L} / \partial_{T}$. As can be understood from expression (7), the determination, as-

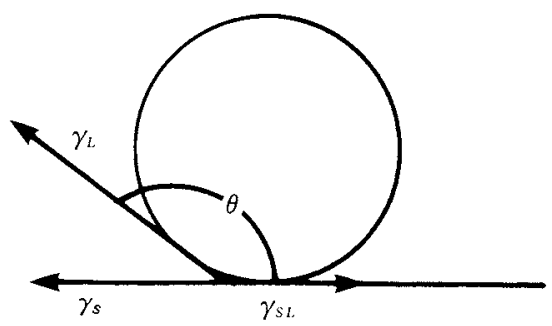

(b)

Fig. 2 Wetting with liquid on a solid surface 
sumes that the angle of contact is constant relative to the temperature $T$.

Meanwhile, the temperature dependency of the surface tension $\left(\partial \gamma_{L} / \partial \gamma_{T}\right)$ can be calculated by using various methods for estimating the properties of constants ${ }^{10)}$.

\section{Wettability measurements}

Table 1 summarizes methods commonly used for determining the wetting of powders.

\section{(1) The heat-of-immersion method}

This method gives the most fundamental values on the wettability of powder. The generation of heat of immersion is attributable to the surface areas of particles, the polarities of particles and liquid, and to the formation of an electrical double layer on the individual particle surface. This method is an important means for analyzing the surface properties of powders. As the method of determining the angle of contact based on the heat of immersion, was previously described, no attempt is made to explain here.

Usually, the magnitude of heat of immersion is considerably small. The resultant temperature level is still low even with inorganic powder having higher energy. Therefore, measuring the heat of immersion of a powder having small specific surface area or a sample with low energy takes significant difficulties or requires long measuring time.

At the cost of inconvenience in measuring, the heat-of-immersion measurement promotes the analysis of the dispersion force caused by the interaction between a powder and liquid, the mechanism of induced dipoles and permanent ones, and the change in the surface conditions of a powder that will occur by heat treatment or chemical reaction.

Recently, Arakawa ${ }^{11)}$, Arai ${ }^{12)}$ and others discussed the correlation between the heat of immersion and the dipole moment, with various powders. Measurement data on the heat of immersion were also disclosed ${ }^{13)}$.

\section{(2) The dripping method ${ }^{14)}$}

The angle of contact with a powder not readily wetted can be measured by packing the powder into a specific formation, placing a droplet onto the powder and directly observing the droplet with a microscope. This technique is theoretically based on the assumption that the liquid does not penetrate into the powder bed. However, in actual operations, measurement can be accomplished after the penetration of the liquid into the powder bed is saturatedly attained.

First, the distance $h$ from the apex of a droplet to the surface of a powder is measured. Using the following expression, the angle of contact $\theta$ is calculated.

$$
\cos \theta=1-\sqrt{\frac{\beta h^{2}}{3(1-\epsilon)}}
$$

Table 1 Methods for measuring the wetting of powders

\section{Method}

The heat-of-immersion method

The dripping method

The capillary method

The liquid phase distribution method

\section{Measuring principle}

Measures the heat of immersion generating when a powder is dispersed in liquid.

A powder is formed into a specific shape onto which a droplet is applied, and the angle of contact is measured with a microscope.

Using a compacted powder bed as composite capillaries, the adhesion tension and angle of contact are determined by the penetration of the liquid medium.

(1) The penetration rate method: wetting is evaluated based on the penetration of the liquid medium.

(2) The pressurizing method: pressure is exerted onto one and of a liquid medium penetration a compacted powder layer. Wetting is evaluated based on the pressure that attains equilibration.

Two liquids not readily blended together are maintained in a dual liquid phase to which powder is added. Wetting is evaluated by observing the migration into the powder. 
where $\beta=\rho_{L} g / 2 \gamma_{L}, \rho_{L}$ represents the density of the liquid, and $\epsilon$ denotes a volumetric void fraction.

Murata $^{15)}$ examined Kossen's formula and gave an expression that is valid under the assumption that capillaries are saturated around a droplet on a compacted powder. He then measured the wettability of a formed coal powder using the equation.

(3) The penetration rate method

Using this method, a cylinder is filled with powder. The angle of contact $\theta$ is then determined by measuring the proceeding rate of the gas-liquid interface formed by the liquid penetrating into the powder layer, or by measuring the change in the amount of the liquid penetrated into the layer.

When a liquid penetrates through the powder bed, the penetration rate can usually be determined by the Hagen-Poiseuille equation as follows:

$$
\frac{\mathrm{d} \ell}{\mathrm{d} t}=\frac{r^{2}}{8 \eta \ell} \Delta p
$$

where $\ell$ represents the distance of penetration, $r$ denotes the average radius of capillaries formed by particles in the powder layer, and $\eta$ is the viscosity of the liquid. Meanwhile, the pressure $\Delta p$ is the addition of the external pressure $\Delta p$ and the tension $\Delta p_{c}$.

The pressure $\Delta p_{c}$ of the capillary tension is defined as:

$$
\Delta p_{c}=\frac{2 \gamma_{L} \cos \theta}{r}
$$

The penetration rate of the powder bed is obtained as:

$$
\frac{\mathrm{d} \ell}{\mathrm{d} t}=\frac{r^{2}}{8 \eta \ell}\left(\frac{2 \gamma_{L} \cos \theta}{r}+\Delta p\right)
$$

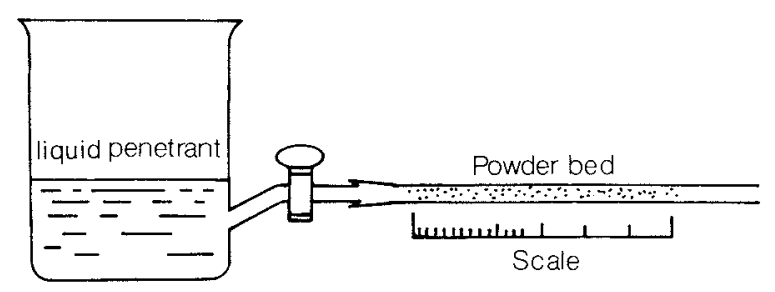

Fig. 3 Apparatus for measuring the penetration rate
Using the penetration rate method, when Eq. (11) is solved under the assumption that the external pressure $\Delta p=0$ and the distance $\ell=0$ at $t=0$ (initial condition of penetration) then the expression is solved:

$$
\ell^{2}=\frac{r \gamma_{L} \cos \theta}{2 \eta} t
$$

Eq. (12) is known as the Washburn equation and has often been applied to measure the penetration rates ${ }^{16)}$.

In this expression, $r$ represents the mean radius of the path (capillaries) in the powder bed. The value $r$ can be estimated using an amount of liquid that completely wets the powder, and by determining the $\ell^{2}$ versus $t$ relationship at $\cos \theta=1$.

In some cases, the value $r$ can be simplified by assuming that the value is half of the average particle size of the powder ${ }^{17,18)}$.

One example of the measuring apparatus in compliance with the Washburn equation is illustrated in Fig. 3. Instead of the visual observation in Fig. 3, Tanaka et al. invented the apparatus in Fig. $4^{19)}$.

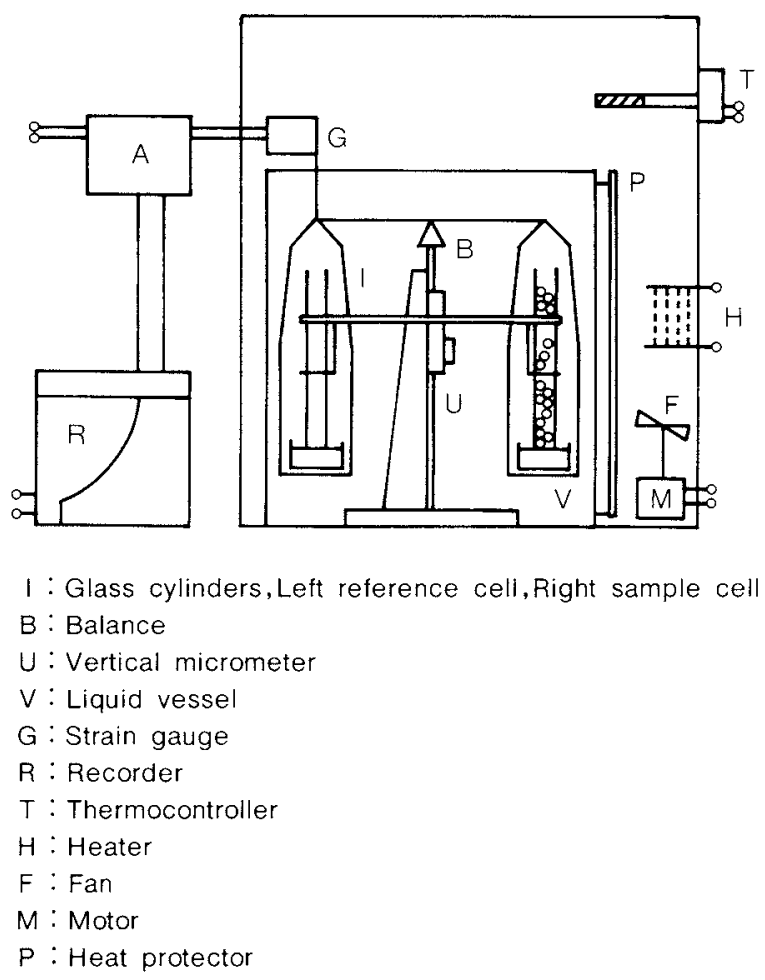

Fig. 4 Apparatus for measuring the penetration rate used by equilibration balance 


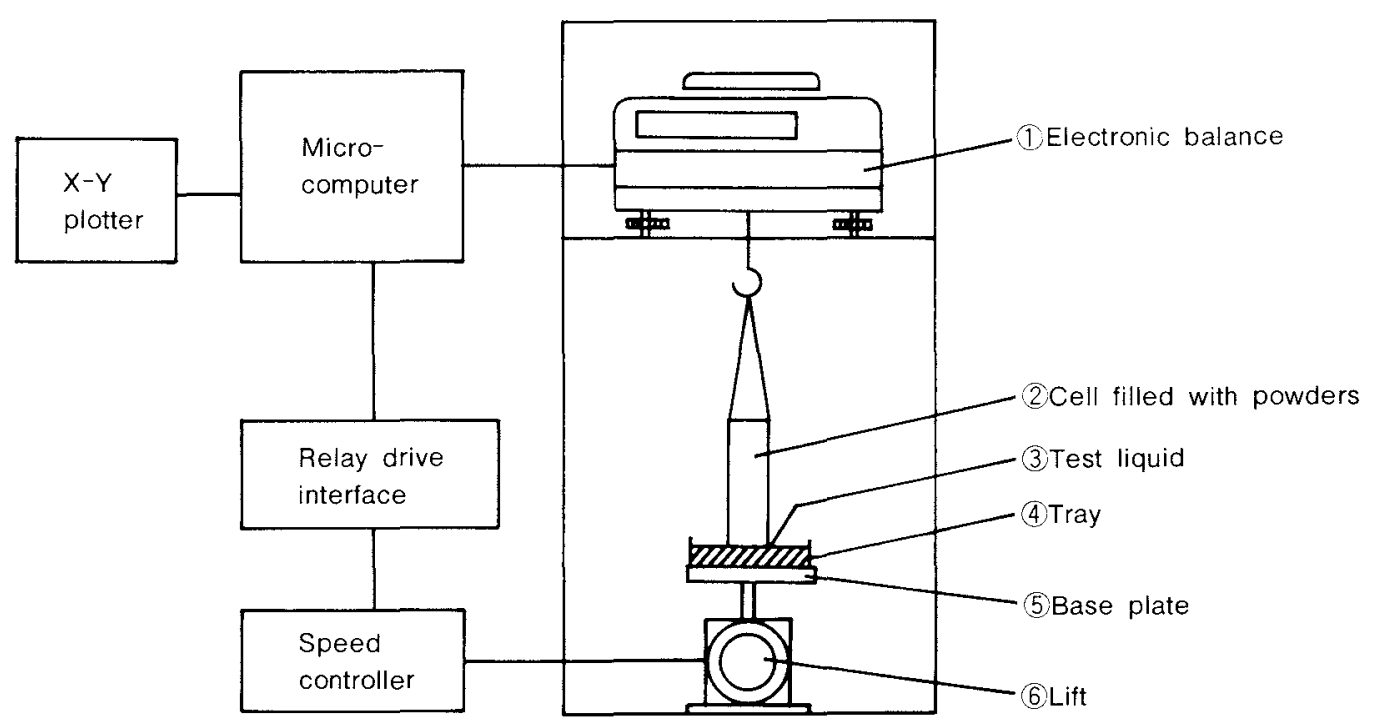

Fig. 5 A measurement apparatus based on electronic balance

Using this apparatus, the powder is poured into one of a pair of cylinders, each having a paper filter on the bottom, while the other cylinder is left empty. A tray filled with a liquid is equilibrated with the other similarly filled tray on the balance. Then the pair of cylinders is allowed to simultaneously come into contact with the liquid. Next, the volume reduction in the liquid caused by liquid penetration into the powder bed is converted into electrical signals by a strain gauge and is recorded for evaluation.

The authors ${ }^{20)}$ developed a measuring ap- paratus employing an electronic balance schematically illustrated in Fig. 5. With this apparatus, a cell filled with a powder bed is suspended from the electronic balance, and a tray filled with test liquid is automatically moved with a lift. A microcomputer detects the contact of the cell with the liquid surface by the change in weight of the cell, and then the microcomputer stops lifting of the cell and automatically measures the weight of the penetrated liquid. The change in the weight of the cell during the operation is summarized in Fig. 6. As shown in this figure, once the cell

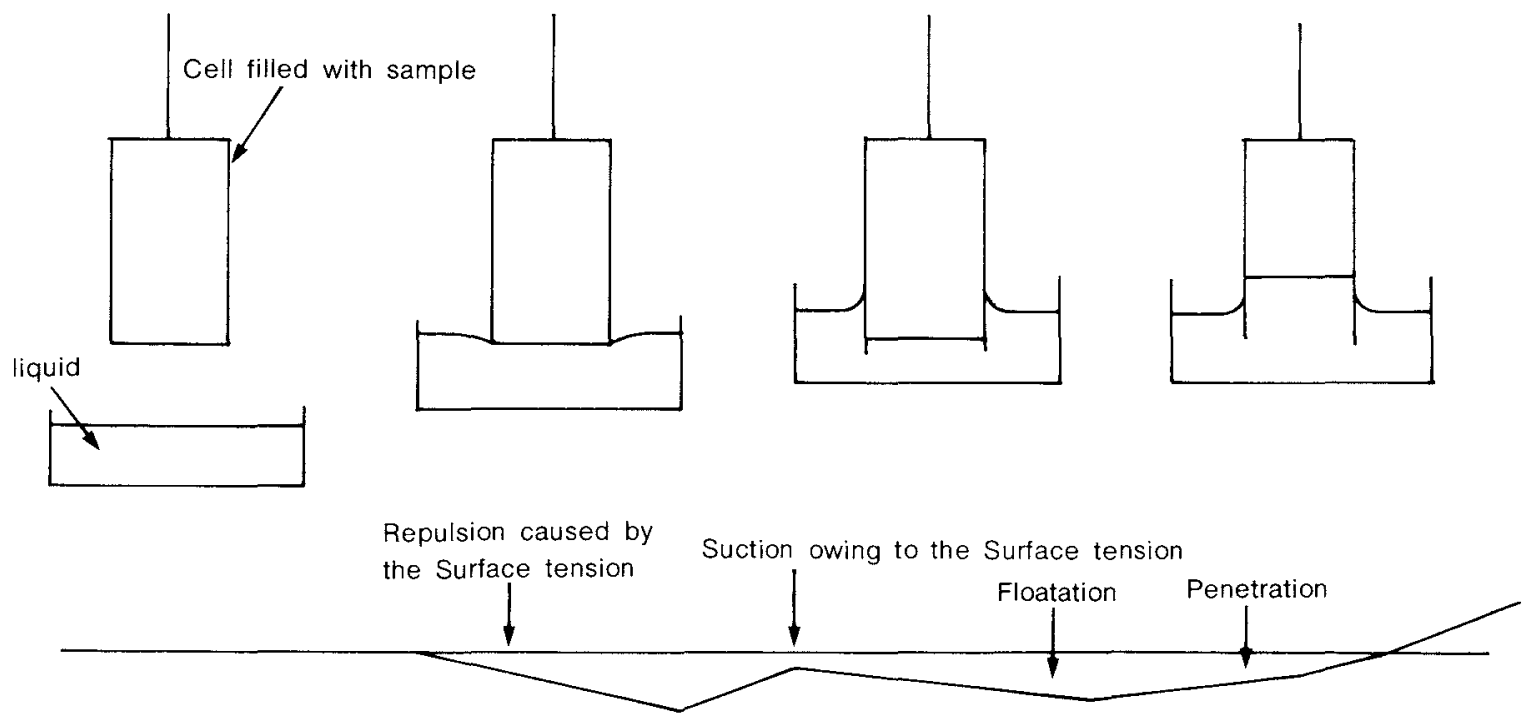

Fig. 6 A method for detecting the contact with liquid surface 


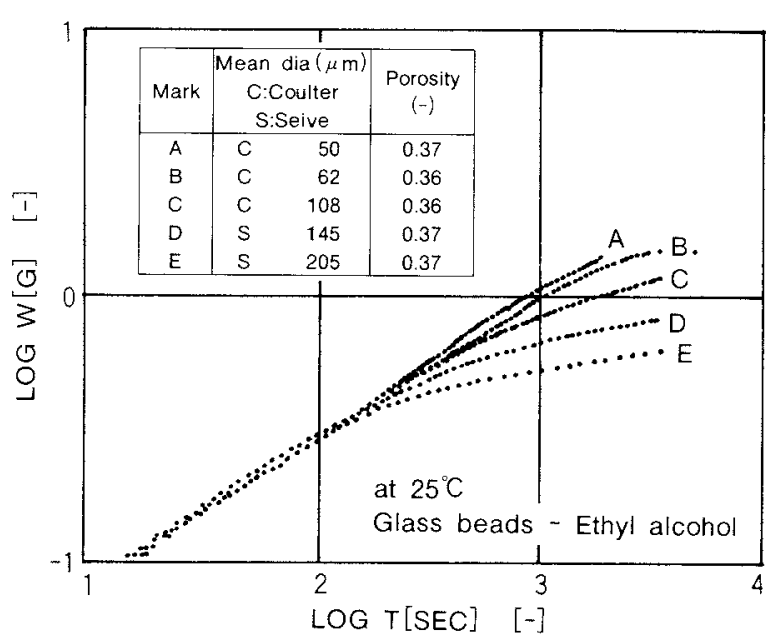

Fig. 7 Weight of ethyl alcohol that penetrated into glass beads

filled with powders comes into contact with the liquid surface, the surface tension of the liquid excludes the cell from the surface. In contrast with this action when the tray is further lifted, the cell receives a suction force by the surface tension, and, then, the cell also receives floatation from the portion immersed in the liquid. After the tray is stopped lifting, the liquid penetrates through the powder layer. The apparent weight of the cell thereby increases again. Sasaki noted that such a penetration process is not limited to powders but is valid even where solid matter is immersed in a liquid ${ }^{21)}$.

The distance of penetration $\ell$ and the weight $W_{L}$ of the liquid penetrated has the following interrelationship:

$$
W_{L}=\epsilon S \ell \rho_{L}
$$

substituting from Eq. (13) in Eq. (15), the relationship is given:

$$
\frac{W_{L}^{2}}{t}=\left(S \epsilon \rho_{L}\right)^{2} \frac{r \gamma_{L} \cos \theta}{2 \eta_{L}}
$$

where $S$ is the cross-sectional area of the powder layer, $\epsilon$ denotes the void fraction, and $\rho_{L}$, $\gamma_{L}$, and $\eta_{L}$ represent the density, surface tension, and viscosity of the liquid, respectively.

Figure 7 shows the results of logarithmic plotting correlated between the weight of penetrated liquid $W_{L}$ and the time $t$. This relationship was obtained by the apparatus shown in Fig. 5, where $W_{L}^{2}$ is apparently proportional to time $t$ for a specific duration. Murata ${ }^{22)} \mathrm{ob}-$

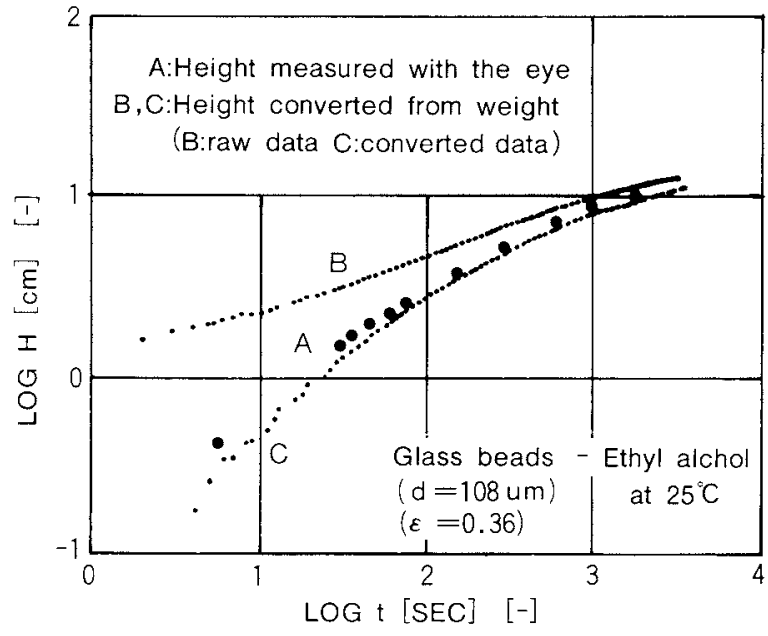

Fig. 8 Comparison of penetrated liquid levels by visual observation and by using the weighing method

tained the similar relationship with both coal powder and $\mathrm{SiO}_{2}$ powder. Using an electronic balance connected with a recorder, Fujitani et al. ${ }^{23)}$, also measured the weight of liquid that penetrated into a glass tube filled with a powder sample.

These methods of measuring the weight of penetration are simple and accurate, whereas conventional methods of visually observing the surface of penetrated liquid are inherently inconvenient and relatively inaccurate. This is because of the irregular rate of the penetration through the powder bed, and because of a greater penetration rate of liquid along the internal wall of the cell filled with powder. The authors ${ }^{24)}$, simultaneously performed a visual inspection and weight-measurement to determine the penetration rate of ethyl alcohol into glass bead layer. The resultant data was plotted in Fig. 8. Once the weights of the cell and paper filter were eliminated from the raw weight data in this figure in order to determine the height of penetrated alcohol, the results were virtually identical for both measuring techniques. However, the results of visual observation were always higher. Therefore, that the penetration rate relative to the inner wall of the cell is always greater than that relative to the powder layer can be deduced from this fact. The authors also found that the liquid traveling along the inner wall affects the scattering of measured data on the penetration rate.

Morishima et al. ${ }^{25)}$ developed a system for measuring the penetration distance by detect- 


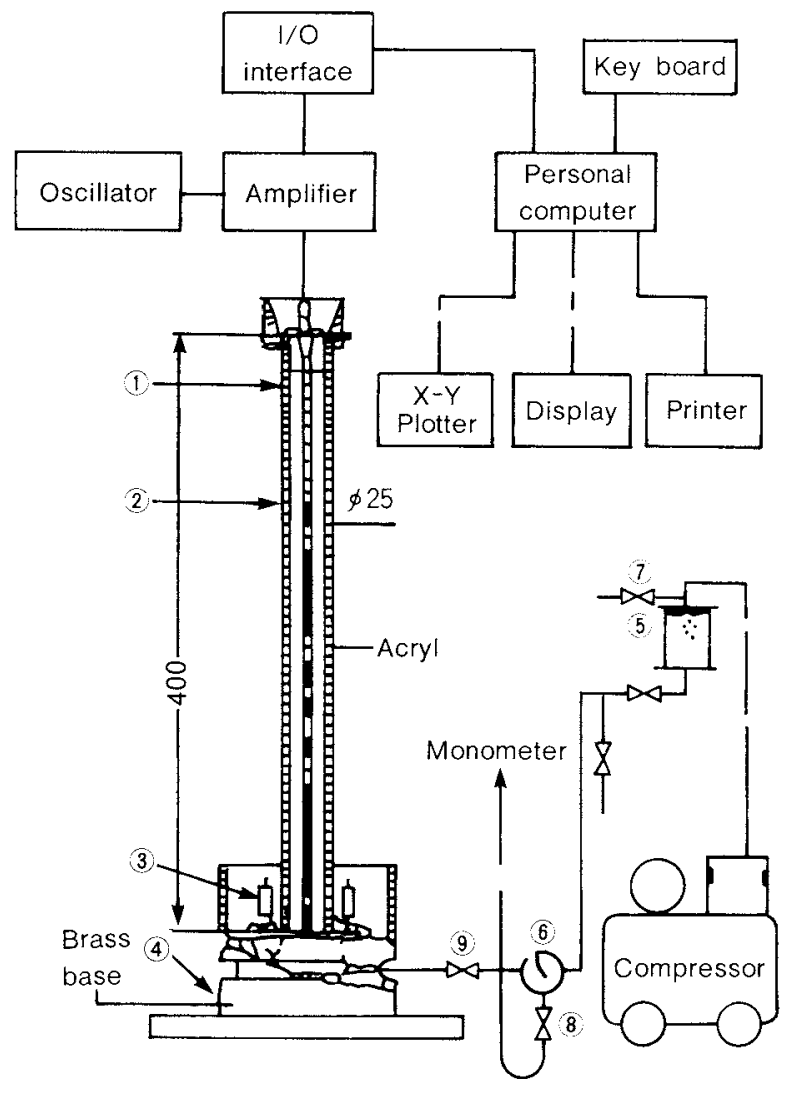

Fig. 9 Schematic diagram of the testing apparatus

ing the electrical conductivity as shown in Fig. 9. In it, electrodes are placed in the cell to determine the vertical position of the penetrated liquid.

Furthermore, Matsumoto et al. developed an apparatus shown in Fig. 10 ${ }^{26)}$. This apparatus also principally uses electrodes for measuring electrical conductivity in order to determine the horizontal position of the penetrated liquid. Optionally, this apparatus can incorporate a photosensor to detect change in the refraction of light caused by the penetrated liquid. This apparatus is advantageous in that a thinner powder layer makes uniform compaction, which means a stable void fraction.

Though simple and easy to operate, the position detection method using an electrode or photosensor has difficulty in measurement. This is because the discontinuous measuring points cannot cope with uneven penetration of the liquid.

(4) The pressurizing method

This type of measuring technique was initially invented by Bartel1 ${ }^{27}$ ), where a cylindrical

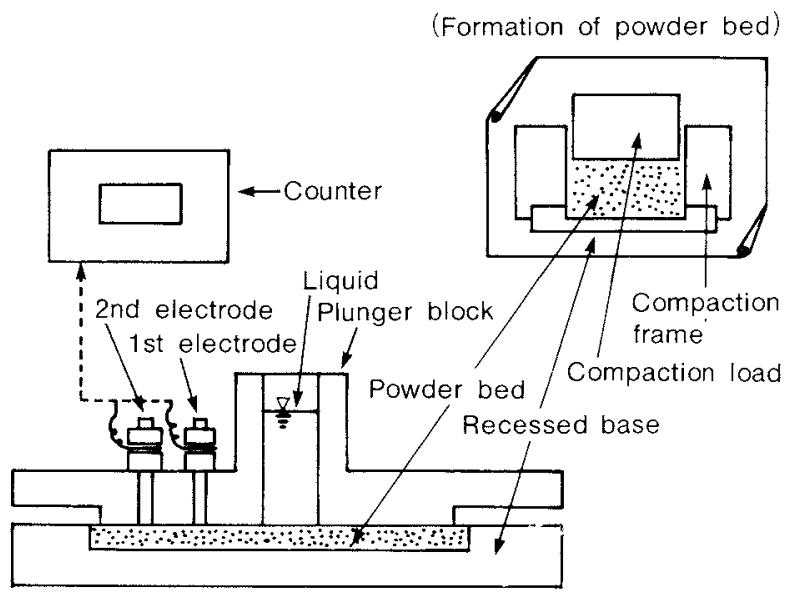

Fig. 10 Apparatus for measuring the horizontal penetration rate

cell filled with powder is placed horizontally. The inside of the cell is depressurized to make liquid to penetrate from one end, while from the other end a pressurized suitable gas is introduced, and then, the cell is sealed off. Next, a pressure $\Delta p$ is determined where the penetration stops and reaches equilibration with the liquid. In this technique, the angle of contact is estimated by Eq. (10).

Though it is difficult to verify the equilibration, the pressurizing method has an advantage. It can be used to evaluate a liquid-liquid system the liquids of which are not readily blended with each other.

Matsumoto et al. $^{28)}$ developed a constant flow rate method, where liquid is supplied into the powder bed at a constant flow rate. At the same time, the pressure applied on the powder bed is measured to determine wettability.

One example of the constant flow rate method is schematically illustrated in Fig. 11. If the evaluated liquid-solid system is that of anhydrous powder and water, the water level in column A is identical to that of column B until the water level reaches plane $0-0^{\prime}$. Once passing through the perforated plate that supports the powder bed, the water temporarily remains stagnant. Then, when the water level in column $B$ reaches a specific point, water again penetrates through the powder layer in column A. Finally, water exceeds the top of the powder layer as shown in Fig. 11 a). In contrast, the pressure pattern of a hydrophilic powder is shown in Fig. 11 b) since water is sucked by the powder bed. 


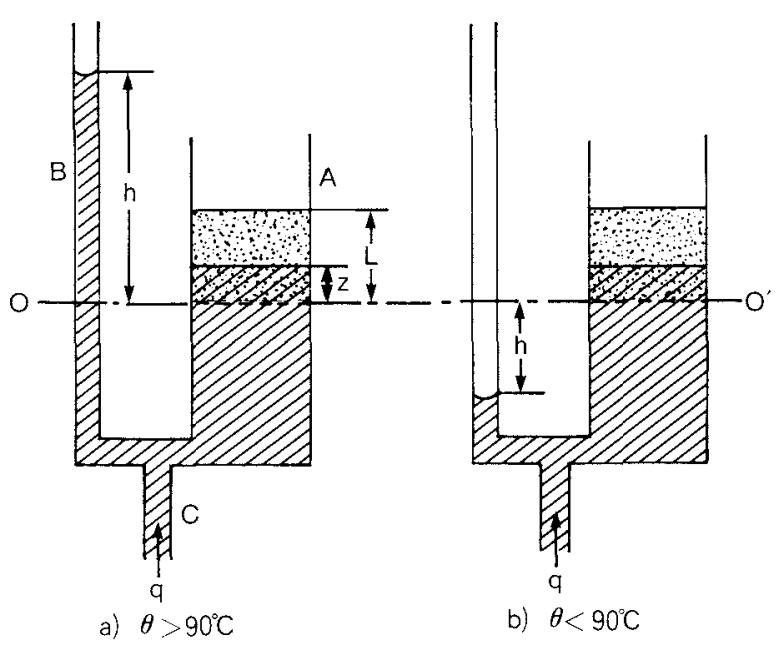

Fig. 11 Schematic of the constant flow rate method (principle)

This technique has limitation with a lyophilic sample in that liquid is rapidly absorbed, and liquid supply is disrupted, giving data with poor replicability. Nevertheless, this technique is regarded as an appropriate means for evaluating the penetration rate of lyophobic powder, the penetration rate of which is otherwise difficult to measure.

\section{Discussion on the penetration rate}

Figure 12 summarizes the penetration rate data measured on various liquids and expressed in weights, using titanium oxide as a powder sample. An angle of contact can be determined using Eq. (14), based both on a linear gradient of this penetration rate diagram, and on theoretical capillary radius determined with the same powder having the same void fraction. In other words, the hydrodynamic radius is determined by the specific surface area measurement using the air permeability method. The resultant angles of contact are not less than $80^{\circ}$, unrealistic values.

The possible reason is that unlike the ideal rapillaries of the straight path, the actual capillaries of the powder bed, which is subjected to the penetration rate measurement, have meandering paths. This must be considered in order to obtain a more realistic angle of contact.

Accordingly, in conjunction with the air permeability method for determining the capillary radius $r$, another method for evaluating the wettability is available. In it, using the penetration rate of a reference liquid with a $\cos \theta=1$

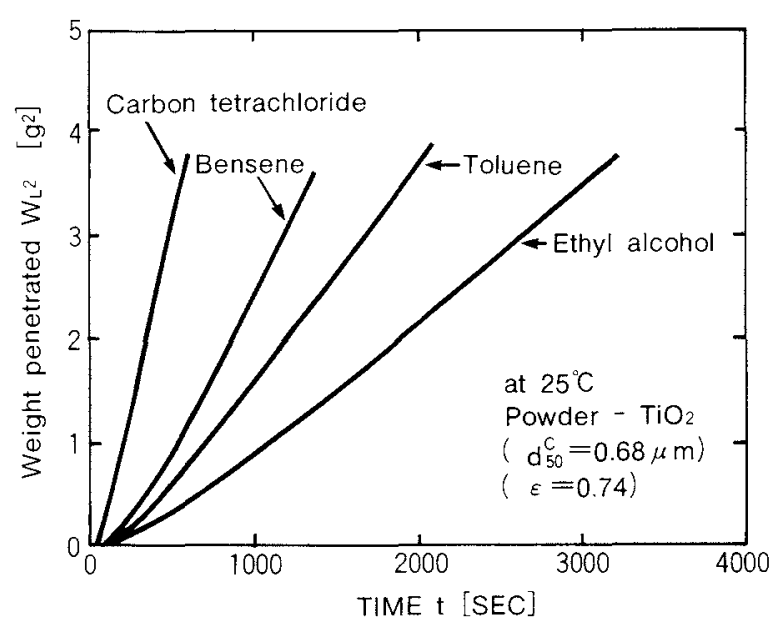

Fig. 12 Some examples of the penetration rate with $\mathrm{TiO}_{2}$

and the penetration rate of the liquid being evaluated, the ratio of the rates is determined in order to evaluate the wettability of the liquid.

For evaluating the penetrations of different liquids into the powder sample, the sample is prepared in the same manner, then the void fraction $\epsilon$ and capillary radius $r$ remain constant. Therefore propsitioning the equations for each cases, the expression reduces to

$$
\frac{\left(W_{L 2}^{2} / t_{2}\right)}{\left(W_{L_{1}}^{2} / t_{1}\right)}=\frac{\rho_{L_{2}}^{2} \eta_{L_{1}} \gamma_{L_{2}} \cos \theta_{2}}{\rho_{L_{1}}^{2} \eta_{L_{2}} \gamma_{L_{1}} \cos \theta_{1}}
$$

The left side of Eq. (15) can be determined by actual measurement, while the density of liquid $\rho_{L}$, viscosity $\eta_{L}$, and surface tension $\gamma_{L}$ can be determined by the literature. The wettability of liquid can thus be evaluated.

Meanwhile, Koishi ${ }^{29)}$ derived the formula based on Poiseuille's equation (9), by incorporating the tortuosity of the capillaries in the powder bed and the fluid friction as follows:

$$
\frac{\mathrm{d} \ell}{\mathrm{d} t}=\frac{r^{2} \rho_{L} g}{8 \eta_{L} k^{2}}\left(\frac{\ell_{\infty}}{\ell_{t}}-1\right)+\frac{r \rho_{L} g}{2 \epsilon k^{2}}\left(\frac{\ell_{\infty}}{\ell_{t}}-1\right)
$$

where $k$ represents a ratio of capillary paths that corresponds to an actual distance and the apparent distance, while $\ell_{\infty}$ is a distance of penetration attained when the equilibration of a penetration rate $\frac{\mathrm{d} \ell}{\mathrm{d} t}=0$ is reached.

Converting Eq. (16) to the weight base, the penetration rate leads to the following expression: 


$$
\frac{\mathrm{d} W_{t}}{\mathrm{~d} t}=K_{1}\left(\frac{W_{\infty}}{W_{t}}-1\right)+K_{2}\left(\frac{W_{\infty}}{W_{t}}-1\right)
$$

where,

$$
K_{1}=\frac{\epsilon S \rho_{L}^{2} g r^{2}}{8 \eta_{L} k^{2}} \quad K_{2}=\frac{\epsilon S \rho_{L}^{2} g r}{2 \epsilon k^{2}}
$$

In Eq. (17), for a larger radius, the first term on the right side alone can be approximated, while for a smaller radius it can be simplified by the second term alone.

Using Eq. (17), Koishi et al. ${ }^{30)}$ plotted $\left(\mathrm{d} W_{t} /\right.$ $\mathrm{d} t)$ and $\left(1 / W_{\ell}\right)$. By the resultant plotting, they determined the coefficients $K_{1}, K_{2}$, and $W_{\infty}$, in order to evaluate the wettability of powder.

There is a problem in the penetration rate method, however. It is that with powder of good wettability, the liquid is rapidly permeated in the particle surface, and the voids in the powder layer are not completely filled with the liquid. Therefore, some voids remain unfilled as bubbles.

Nakagawa et a1. ${ }^{31)}$ studied this phenomenon by evaluating the void structures in powder bed.

\section{The evaluation of surface properties}

To improve dispersion in liquid, $\mathrm{SiO}_{2}$ or $\mathrm{TiO}_{2}$ powder is often subjected to a physical adsorption technique where a compound having an oil-attracting radical is allowed to be fixed onto the particles. Also, a surface treatment technique may be used where the surfaces of the particles are modified by a chemical reaction.

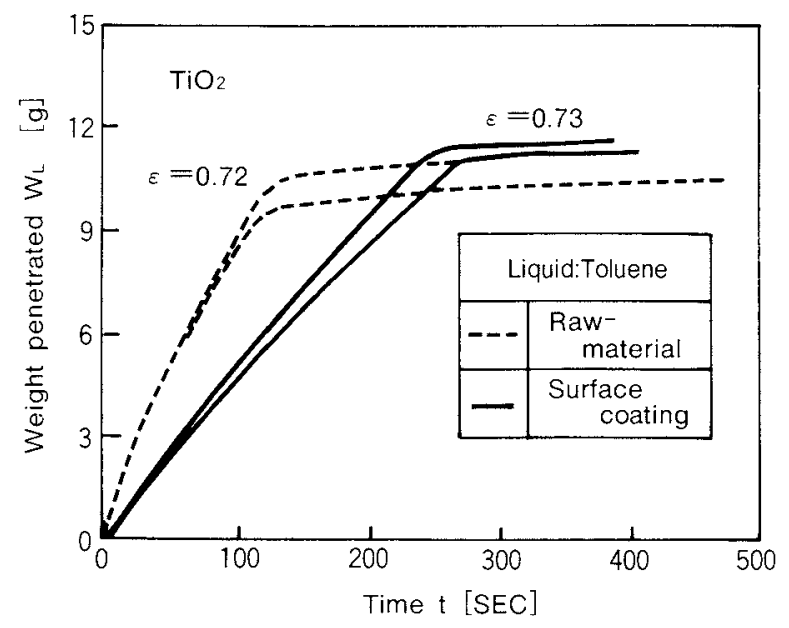

Fig. 13 Effect of the surface coating
For instance, it is known ${ }^{32)}$ that once polyvinyl alcohol (PVA) is adsorbed onto the surface of $\mathrm{SiO}_{2}$ particles, the $\mathrm{OH}$ group on the $\mathrm{SiO}_{2}$ surface is strongly bonded to the $\mathrm{OH}$ group of the side chain on the PVA, hydrophobic hydrocarbon groups are thereby oriented on the particle surface, and the $\mathrm{SiO}_{2}$ surface become hydrophobic.

Figure 13 indicates the example of the evaluation on the effects of the surface modification. The data was obtained by the penetration rate measurement ${ }^{33)}$.

The powder samples used had a virtually identical particle size distribution and void fraction, where the effects of the surface modification were evaluated. As shown in the electromicrographs of Fig. 14, the surface of individual $\mathrm{TiO}_{2}$ particles modified with alumina $\mathrm{Al}_{2} \mathrm{O}_{3}$, etc. has minute ruggedness compared to that of non-modified particles. The specific surface area by the air-permeability method revealed that the modified particles had a surface area four times as large as that of unmodified particles. It seems that such a change in surface properties of the particles affected the data the resulted from penetration rate measurement.

Recently, methods of combining different types of powders in order to develop new materials has gained researchers' attention. One such method is known as the "mechanofusion system"34).

According to this method, the extremely powerful mechanical energy of "Angmill", which is an ultrafine milling machine using grinding and shearing forces as an operational principle, makes the surfaces of the particles to be fused with another material in order to obtain particles that have unconventional physical properties.
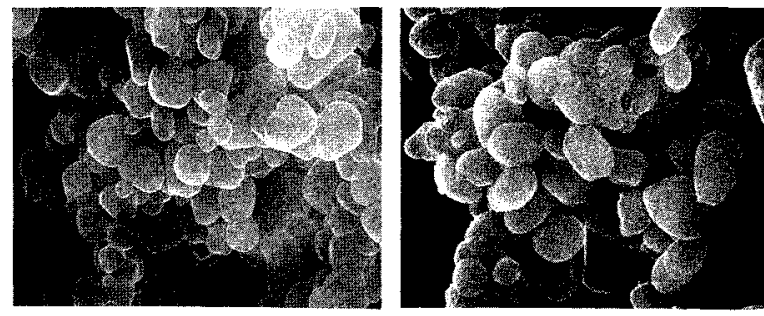

Titaninm ox;de untreated

Fig. 14 Photomicrographs of the surface of titan dioxide samples 


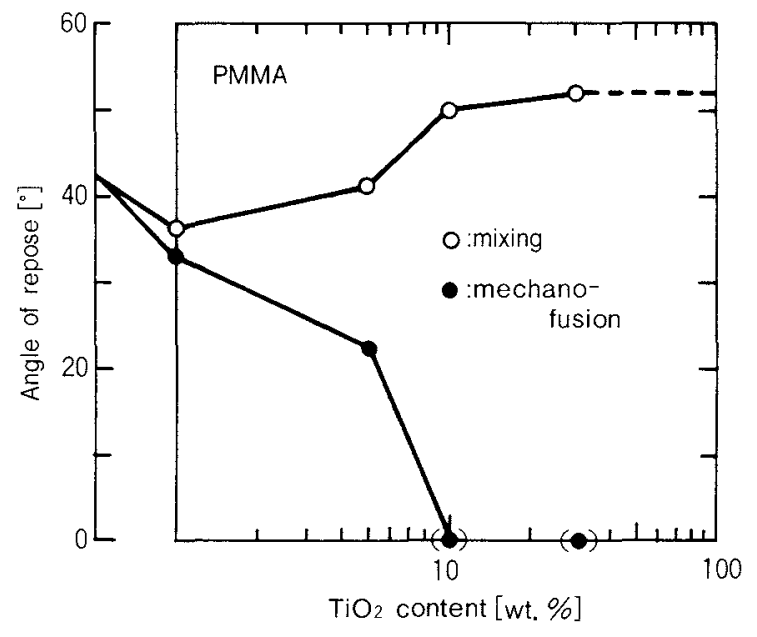

Fig. 15 The mixing ratio and angle of repose of $\mathrm{TiO}_{2}$

Once fused, the resultant particles have surface properties different from those of the original powder materials. The penetration rate method has capable of evaluating this change.

Figure 15 illustrates the variation in the angle of repose, where one sample type was prepared by fusing $\mathrm{TiO}_{2}$ particles onto PMMA particles. The other sample was prepared by mixing. A smaller angle of repose means better fluidity of powder, and this relationship is comparable to the previously mentioned relationship. The smaller angle of contact shows that a liquid medium has better affinity with a solid. The mechanofused type of sample exhibits better fluidity that corresponded to the content of added $\mathrm{TiO}_{2}$ than a non-modified parent material lacking $\mathrm{TiO}_{2}$ particles. The angle of repose reached $0^{\circ}$ with $10 \mathrm{wt} \%$ of $\mathrm{TiO}_{2}$ added. In contrast, the mixture type of sample showed the smallest angle of repose with $1 \mathrm{wt} \%$ of $\mathrm{TiO}_{2}$ mixed. The angle of repose of this type of sample became again greater with a larger ratio of $\mathrm{TiO}_{2}$ mixed, the pattern showed different from that of the mechanofusionmodified type of sample.

Figure 16 illustrates the results of the penetration rates of water into the mechanofusionmodified type of sample. The mother material, PMMA, has no water penetration. However, the larger the rate of $\mathrm{TiO}_{2}$ added was, the larger the penetration rate became. The individual samples used, however, had different void fractions. Therefore, correction had to be incorporated.

Accordingly, taking the previously described

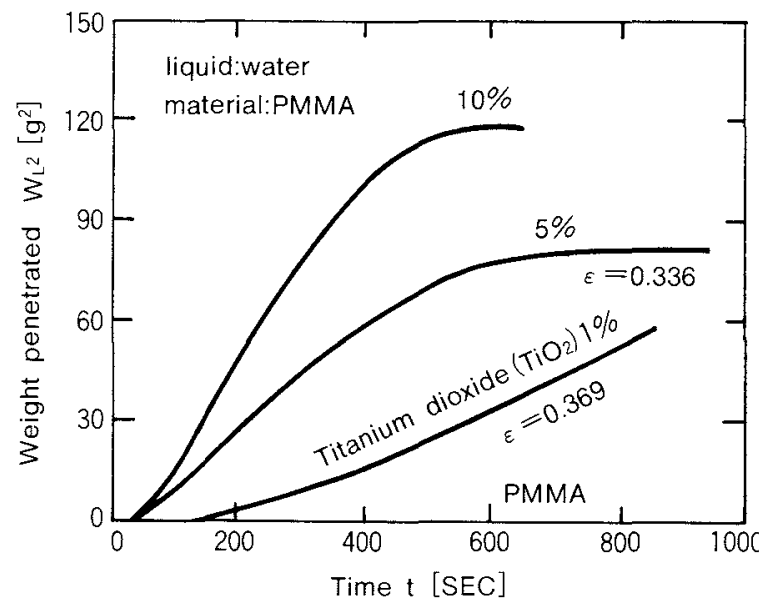

Fig. 16 The penetration ratio measurement with mechanofusion-processed materials

ratio of the Washburn equation, and using, as reference, the data of the mechanofused sample $(1 \mathrm{wt} \%)$, the data listed in Table 2 was obtained. Obviously, the larger the rate of $\mathrm{TiO}_{2}$ added, the greater the wettability of the sample and the smaller the angles of contact.

Figure 17 illustrates electromicrographs of unmodified PMMA particles, and of mechanofusion-modified ( $1 \mathrm{wt} \% \mathrm{TiO}_{2}$ particles) PMMA particles, respectively.
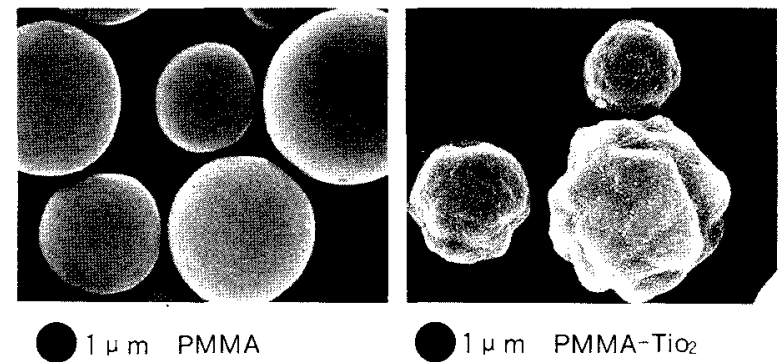

Fig. 17 Mechanofusion of PMMA particles

Table 2 Evaluation of mechanofused materials for wettability

\begin{tabular}{|c|c|c|c|}
\hline Sample & & $\frac{\cos \theta}{(\cos \theta) 1 \%}$ & 要 \\
\hline $\begin{array}{l}\text { Mechanofusion- } \\
\text { processed material }\end{array}$ & $1 \%$ & 1 & $\frac{0}{\Delta 00}$ \\
\hline Same as above & $5 \%$ & 1.52 & 1 \\
\hline Same as above & $10 \%$ & 3.09 & 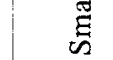 \\
\hline
\end{tabular}




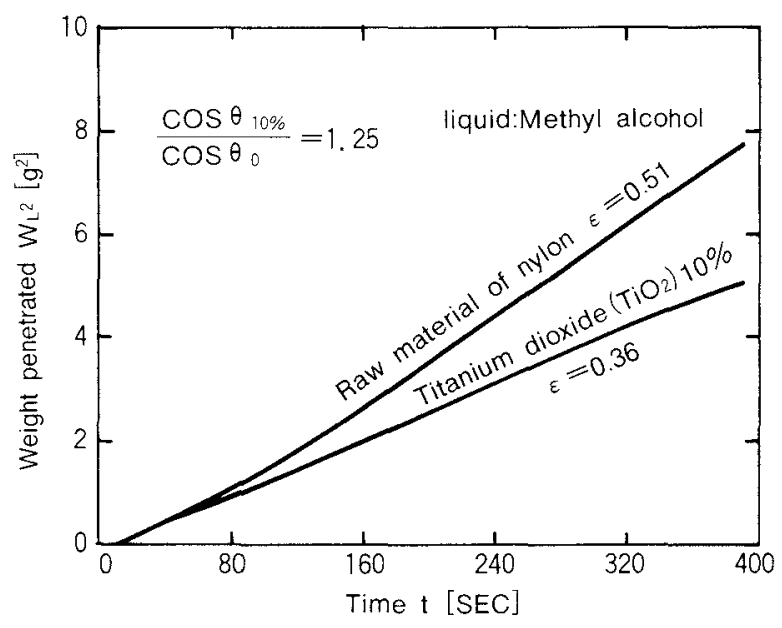

Fig. 18 The penetration rate of ordinary nylon and that of nylon the surface of which is being fused with titanium dioxide

Figure 18 indicates the penetration rates of methyl alcohol into nylon particles having mechanofusion treatment with $\mathrm{TiO}_{2}$ particles on the surface of the nylon particles, and unmodified one. In this figure, the penetration rate with the unmodified nylon particles is greater than that of mechanofusion modified sample. Since nylon powder had a larger void fraction than that of $\mathrm{TiO}_{2}$ particles, it was impossible to directly evaluate the affinity of methyl alcohol and nylon.

To solve this problem, the same evaluation method as used for the previously mentioned PMMA particles was performed. Thus, void fractions of the respective sample were sub-

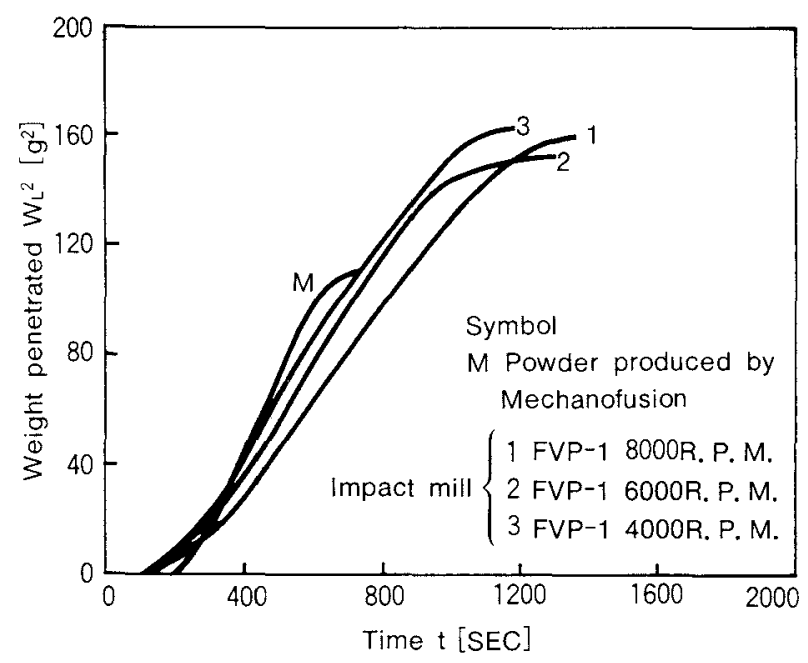

Fig. 19 Penetration ratios of PMMAs the surface of which is fused respectively with different methods
Table 3 Comparison of wettability treated by mechanofusion and impact mill

\begin{tabular}{c|c|c} 
Preparation method & Conditions & $\cos \theta / \cos \theta_{8000}[-]$ \\
\hline \multirow{2}{*}{$\begin{array}{c}\text { Impact mill } \\
\text { FVP }\end{array}$} & 8000 r.p.m. & 1 \\
\hdashline 6000 & 0.99 \\
& 4000 & 1.24 \\
\hline \multicolumn{2}{|c}{ Mechanofusion } & 1.91 \\
\hline
\end{tabular}

stituted in the Washburn equation in order to determine the ratio between the two samples. The mechanofusion-modified particles have good wettability with methyl alcohol compared to the unmodified nylon sample.

An another method for obtaining a new material without the mechanofusion technique, is an application of a mechanical impact mill. Figure 19 illustrates the penetration rate measurement data obtained by various pulverization conditions with a fine-micron mill this is one type of the impact mill, as well as the corresponding data of the modified material by the mechanofusion.

Obtained by this penetration rate measurement, the void fraction and particle size were corrected using relative comparison based on the Washburn equation. The result is summarized in Table 3.

On the affinity with the powder and water conditions 1 and 2 is deemed identical with respect to fluctuation in measured values. Affinity is better with condition 3 , and the mechanofusion modified sample has the best affinity.

With the samples treated with the impact mill, and in particular with samples on the condition of 8,000 and $6,000 \mathrm{rpm}$, fusion onto the parent material seems to be attributable primarily to impact; while a sample treated at $4,000 \mathrm{rpm}$, includes frictional crushing mechanism marginally so that affinity is improved than those of higher rotations.

The mechanofusion process seems to involve not only impact but also strong friction or frictional grinding. It appears that the frictional grinding makes ultrafine particles to firmly deposit onto the surface of the parent material, and the interaction between the coarser particles and ultrafine particles generates. Thus, "fusion" acts on the individual particle-particle 
interface.

For this mechanism, applying mechanofusion to various powders, whether they be organic, inorganic, or metallic, can create novel materials.

\section{Conclusion}

In this paper, the technique for evaluating wettability with powders in Japan has been summarized. Penetration rate measurement techniques were especially focused on.

Wettability evaluation is an important technique not only in conventional powder dispersion in liquid but also in the creation of composite particles $^{35)}$ which has been attracting the attention of researchers recently.

Mounting attention is being given to the wettability of powder. This is a continuous source of topics in various conferences and seminar meetings ${ }^{36}$ ).

It is hoped that this report helps promote further research on this theme.

\section{References}

1) D.J. Show: Introduction to Colloid and Surface Chemistry, 3rd. Ed., Butterworths (1980) Translated into Japanese by F. Kitahara and K. Aoki, Hirokawa Shoten (1983).

2) M. Nakagaki: "Hyomenjotai to Koroidojotai", Tokyo-Kagaku-Dozin (1969).

3) Jpn. Chemical Society: "Shin Jikken Kagaku Kouza 18, "Kaimen to Koroido", Maruzen (1977).

4) E.W. Washburn: Phys. Rev., 17, 273 (1921).

5) R.L. Peek and D.A. McLean: Ind. Eng. Chem. Anal Ed., 8, 85 (1934).

6) H. Sanuki: Fiber Engineering, 24, 467 (1971).

7) H. Sanuki: Ibid. ,25, 496 (1972).

8) K. Kurematsu: "Zairyo Gijutsu", 5, 34 (1987).

9) M. Koishi and M. Tsunoda: "Funtai no Hyomen Kagaku”, Nikkan-Kogyo-Shimbun (1975).

10) K. Sato: "Bussei Teisu Suizanho", Maruzen (1954).

11) M. Arakawa: "Saishin Funtai no Zairyo Sekkei", Tekuno shisutemu (1988).

12) Y. Arai: "Funtai no Zairyo Kagaku", Baifukan (1987).

13) H. Murayama and K. Meguro: J. Japan Soc. Colour Mat., 43, 461 (1970).
14) N.W. Kossen and P.M. Heertjes: Chem. Eng. Sci., 20, 593 (1955).

15) T. Murata: "Funtai to Kogyo", No.4, 26 (1981).

16) H. Kuno and R. Abe: Ind. Chem. 61, 1445 (1958).

17) Ibid., 11).

18) M. Nishino and M. Arakawa: J. Soc. Mat. Sci., Japan, 22, 663 (1973).

19) Y. Tanaka and M. Koishi: J. Japan Soc. Colour Mat., 49, 22 (1976).

20) H. Honda, Y. Taniguchi, T. Ishizaka, T. Yokoyama, N. Kaya, T. Matsuno and M. Koishi: "Zairyo Gijutsu", 3, 489 (1985).

21) N. Sasaki: "Zairyo Gijutsu", 5, 298 (1987).

22) T. Murata: J. Japan Mining Soc., 98, (1982).

23) T. Fujita and T. Sato: J. Japan Soc. Colour Mat., 55, 459 (1982).

24) T. Yokoyama and N. Kaya: Preprints of 1984 Autumn Research Presentation, p. 13 (1984).

25) N. Morishima, K. Maehara and Y. Nomura: Preprints of 1983 Autumn Research Presentation, p. 40 (1983)

26) K. Matsumoto, Y. Tabata, G. Hong and K. Watanabe: J. Soc. Powder Tech., Japan, Soc. 25, 365 (1988).

27) F.E. Bartell, L.S. Bartell: J. Am. Chem. Soc., 56, 2208 (1934).

28) K. Matsumoto, H. Akutsu, A. Yoshimidzu, G. Hong and K. Watanabe: J. Soc. Powder Tech., Japan, Soc., 25, 359 (1988).

29) M. Koishi and Y. Tsuriya: "Bunsan Gijutsu Nyumon", Nikkan-Kogyo-Shimbunsha (1972).

30) Y. Tanaka and M. Koishi: J. Japan, Soc. Colour Mat., 49, 473 (1976).

31) M. Nakagawa, M. Furuuchi, K. Miwa and G. Gotoh: J. Soc. Powder Tech., Japan of Pow. Tech. Soc., 23, 845 (1986).

32) Y. Arai: "Funtai no Zairyo Kogaku", Baifukan (1987).

33) M. Koishi, N. Kaya, T. Yokoyama and H. Kitai: Preprints of the 24th Discussion on Powder, p. 182 (1983).

34) T. Yokoyama, K. Urayama, M. Naito, M. Kato and T. Yokoyama: KONA [5], 59 (1987).

35) H. Honda, T. Matsuno and M. Koishi: "Zairyo Gijutsu", 6, 186 (1988).

36) M. Koishi, K. Kurematsu, M. Kuwa and N. Kaya: "Ganshin Gijutsu to sono Ouyo" Seminar, Manuscripts, Tekuno Shisutemu (1988). 